\title{
MAGLEV WINDMILL
}

\author{
Minu John ${ }^{1}$, Rohit John ${ }^{2}$, Syamily P.S ${ }^{3}$, Vyshak P.A ${ }^{4}$ \\ ${ }^{1}$ Student, Dept. of EEE, Mar Athanasius College of Engineering, Kothamangalam, India \\ ${ }^{2}$ Student, Dept. of EEE, Mar Athanasius College of Engineering, Kothamangalam, India \\ ${ }^{3}$ Student, Dept. of EEE, Mar Athanasius College of Engineering, Kothamangalam, India \\ ${ }^{4}$ Student, Dept. of EEE, Mar Athanasius College of Engineering, Kothamangalam, India
}

\begin{abstract}
Magnetic levitation, maglev, or magnetic suspension is a method by which an object is suspended with no support other than magnetic fields. Magnetic pressure is used to counteract the effects of the gravitational and any other accelerations. The principal advantage of a maglev windmill from a conventional one is, as the rotor is floating in the air due to levitation, mechanical friction is totally eliminated. That makes the rotation possible in very low wind speeds. Maglev wind turbines have several advantages over conventional wind turbines. For instance, they're able to use winds with starting speeds as low as 1.5 meters per second (m/s). Also, they could operate in winds exceeding $40 \mathrm{~m} / \mathrm{s}$.
\end{abstract}

Keywords: Wind Energy, Magnetic Levitation, Power Generation, Magnets

$* * *$

\section{INTRODUCTION}

Wind is a form of solar energy. It is a natural power source that can be economically used to generate electricity. The way in which wind is created is from the atmosphere of the sun causing areas of uneven heating. In conjunction with the uneven heating of the sun, rotation of the earth and the rockiness of the earth's surface winds are formed. The terms wind energy or wind power describes the process by which the wind is used to generate mechanical power or electricity. Wind turbines convert the kinetic energy in the wind into mechanical power. This mechanical power can be used for specific tasks (such as grinding grain or pumping water) or a generator can convert this mechanical power into electricity. The wind turbine is used for conversion of kinetic energy of wind into electrical energy. The wind turns the blades, which spin a shaft, which connects to a generator and makes electricity.

The Maglev wind turbine design is a vast departure from conventional propeller designs. Its main advantages are that it uses frictionless bearings and a magnetic levitation design and it does not need to vast spaces required by more conventional wind turbines. It also requires little if any maintenance. The Maglev wind turbine was first unveiled at the Wind Power Asia exhibition in Beijing 2007. The unique operating principle behind this design is through magnetic levitation. Magnetic levitation is supposedly an extremely efficient system for wind energy. The vertically oriented blades of the wind turbine are suspended in the air replacing any need for ball bearings.

\subsection{Need for Wind Power Technology}

Renewable energy is generally electricity supplied from sources, such as wind power, solar power, geothermal energy, hydropower and various forms of biomass. These sources have been coined renewable due to their continuous replenishment and availability for use over and over again. The popularity of renewable energy has experienced a significant upsurge in recent times due to the exhaustion of conventional power generation methods and increasing realization of its adverse effects on the environment. It is estimated that renewable sources might contribute about $20 \%-50 \%$ to energy consumption in the later part of the 21st century. Facts from the World Wind Energy Association estimates that by 2010, 160GW of wind power capacity is expected to be installed worldwide which implies an anticipated net growth rate of more than $21 \%$ per year. Maglev wind turbines have several advantages over conventional wind turbines. For instance, they're able to use winds with starting speeds as low as 1.5 meters per second $(\mathrm{m} / \mathrm{s})$. Also, they could operate in winds exceeding $40 \mathrm{~m} / \mathrm{s}$. It would also increase generation capacity by $20 \%$ over conventional wind turbines and decrease operational costs by $50 \%$. This makes the efficiency of the system higher than conventional wind turbine. Currently, the largest conventional wind turbines in the world produce only five megawatts of power. However, one large maglev wind turbine could generate one GW of clean power, enough to supply energy to 75,000 homes ${ }^{[1]}$. The turbine uses permanent type of rare earth magnets (neodymium) instead of electromagnets and therefore it doesn't require electricity to run. The friction between the turbine blades and the base can maximum power output. 


\section{WIND POWER \& WIND TURBINES}

\subsection{Wind Power Technology}

Wind power technology is the various infrastructure and process that promote the harnessing of wind generation for mechanical power and electricity. This basically entails the wind and characteristics related to its strength and direction, as well as the functioning of both internal and external components of a wind turbine with respect to wind behavior. Wind power, as an alternative to fossil fuels, is plentiful, renewable, widely distributed, clean, produces no greenhouse gas emissions during operation and uses little land. Any effects on the environment are generally less problematic than those from other power sources. 83 countries around the world are using wind power on a commercial basis. Worldwide there are now over two hundred thousand wind turbines operating, with a total nameplate capacity of 238,351 MW as of end 2011.

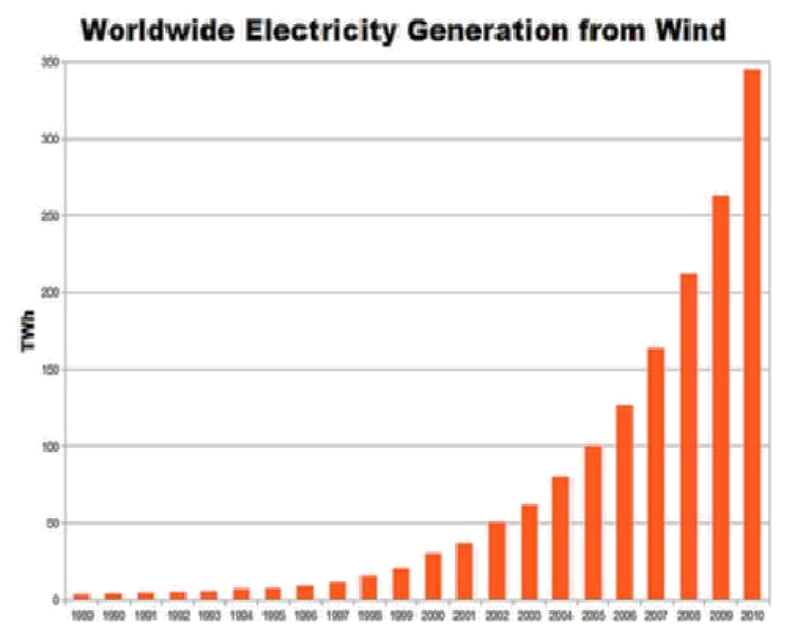

Fig 2.1 - Electricity generation from wind

\subsection{The Power of Wind}

As mentioned earlier the effective functioning of a wind turbine is dictated by the wind availability in an area and if the amount of power it has is sufficient enough to keep the blades in constant rotation. The wind power increases as a function of the cube of the velocity of the wind and this power is calculable with respect to the area in which the wind is present as well as the wind velocity. When wind is blowing the energy available is kinetic due to the motion of the wind so the power of the wind is related to the kinetic energy.

We know,

$$
\text { Kinetic Energy =1/2 MV2 }
$$

The volume of air passing in unit time through an area A, with speed $\mathrm{V}$ is $\mathrm{AV}$ and its mass $\mathrm{M}$ is equal to the Volume $\mathrm{V}$ multiplied by its density $\rho$ so:

$$
M=\rho \mathrm{AV}
$$

Substituting the value of $\mathrm{M}$ in equation above we get:

$$
\begin{gathered}
\text { Kinetic Energy }=1 / 2(\rho A V) \text { V2 } \\
\text { Kinetic Energy=1/2 } \rho \text { AV3 }
\end{gathered}
$$

To convert the energy to kilowatts, a non-dimensional proportionality constant $\mathrm{k}$ is introduced where,

$$
\mathrm{K}=2.14 \times 10-3
$$

Therefore,

$$
\text { Power in KW }(\mathrm{P})=2.14 \rho \mathrm{AV} 3 \times 10-3
$$

Where:

Air Density $(\rho)=1.2 \mathrm{~kg} / 3 / 2.33 \times 10-3$ slugs $/ \mathrm{f} 3$

Area $(\mathrm{A})=$ Area swept by the blades by the turbine

Velocity $(\mathrm{V})=$ wind speed in $\mathrm{m} / \mathrm{s}$

With the above equation, the power being generated can be calculated, however one should note that it is not possible to convert all the power of the wind into power for generation.

The power harnessed from the wind cannot exceed $59 \%$ of the overall power in the wind. Only a portion can be used and that usable portion is only assured depending on the wind turbine being used and the aerodynamic characteristics that accompany it.

\subsection{Types of Wind Turbines}

Many types of turbines exist today and their designs are usually inclined towards one of the two categories: horizontal-axis wind turbines (HAWTs) and vertical-axis wind turbines (VAWTs). As the name pertains, each turbine is distinguished by the orientation of their rotor shafts. The former is the more conventional and common type everyone has come to know, while the latter due to its seldom usage and exploitation, is quiet unpopular. The HAWTs usually consist of two or three propeller-like blades attached to a horizontal and mounted on bearings the top of a support tower. When the wind blows, the blades of the turbine are set in motion which drives a generator that produces AC electricity. For optimal efficiency, these horizontal turbines are usually made to point into the wind with the aid of a sensor and a servomotor or a wind vane for smaller wind turbine applications. With the vertical axis wind turbines, the concept behind their operation is similar to that of the horizontal designs.

The major difference is the orientation of the rotors and generator, which are all vertically arranged, and usually on a shaft for support and stability. This also results in a different response of the turbine blades to the wind in 
relation to that of the horizontal configurations. Their design makes it possible for them to utilize the wind power from every direction unlike the HAWTs that depend on lift forces from the wind similar to the lift off concept of an airplane. Vertical axis wind turbines are further subdivided into two major types namely the Darrieus model and the Savonius model. Darrieus Model which was named after designer and French aeronautical engineer, Georges Darrieus. This form of this design is best described as an eggbeater with the blades, two or three of them bent into a c-shape on the shaft. Finnish engineer Sigurd Savonius invented the Savonius model. The functioning of this model is dependent on drag forces from the wind. This drag force produced is a differential of the wind hitting by the inner part of the scoops and the wind blowing against the back of the scoops. Like the Darrieus model, the Savonius turbines will work with winds approaching in any direction and also work well with lower wind speeds due to their very low clearance off the ground.

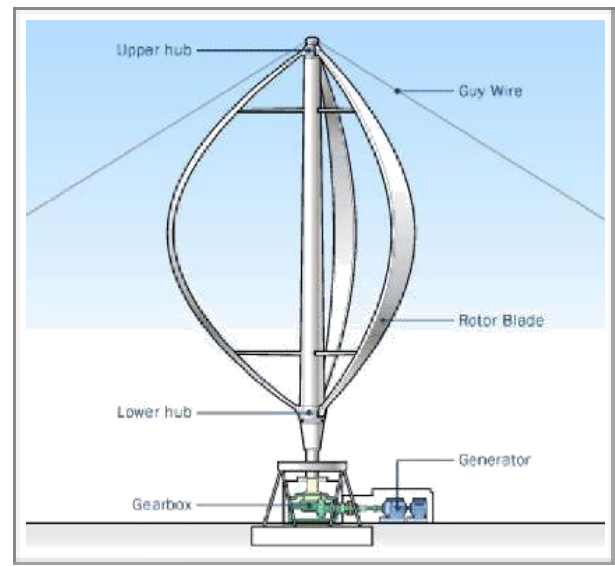

Fig 2.3 - Vertical Axis Turbine

\subsection{Major Components \& Operation of Wind Turbine}

A wind turbine basically draws the kinetic energy from the wind and converts this power to electrical energy by means of a generator. Its operability is dependent on key components of the turbine and its response to the wind based on how it is built. Figure 2.5 shows an illustration of a conventional wind turbine and its parts. With this turbine, the blades receive the wind and are caused to lift and rotate. Depending on the wind speed the controller will start up or shut off the turbine. If wind speeds are right between 8 to 16 miles per hour, the turbine would start to operate but will shut down if speeds exceed about 55 miles per hour. This is a preventative measure because at very high winds the turbine could be damaged. The anemometer on the turbine calculates this wind speed and sends the information to the controller.

The VAWTs usually do not have anemometers because they are usually used for low speed and small scale applications. The high speed shaft drives the generator to produce electricity and they are connected to the low speed shaft by gears to increase their rotational speed during operation. Most generators usually require a rotational speed of about 1000 to 1800 rotations per minute so the gears increases them significantly from 30 to 60 rotations per minute to the electricity producing threshold. All these components sit on a tower usually made out of steel or concrete. The height of the tower is dependent on the size of the rotors and the desired amount of electricity generation. Taller towers serve as an advantage because wind speed is abundant with height so the rotors will work well with increased tower height and promote more and efficient electricity generation.

\section{THE WINDMILL}

This section introduces and provides a brief description of the major components and factors that will contribute to an efficiently functioning wind turbine. These factors are wind power, the generator, magnetic levitation. Later sections will provide an in-depth look into the essence of each factor and its function and importance to the overall operation of the vertical axis wind turbine.

\subsection{Wind Power}

Undoubtedly, the project's ability to function is solely dependent on the power of wind and its availability. Wind is known to be another form of solar energy because it comes about as a result of uneven heating of the atmosphere by the sun coupled with the abstract topography of the earth's surface. With wind turbines, two categories of winds are relevant to their applications, namely local winds and planetary winds. The latter is the most dominant and it is usually a major factor in deciding sites for very effective wind turbines especially with the horizontal axis types.

These winds are usually found along shore lines, mountain tops, valleys and open plains. The former is the type you will find in regular environments like the city or rural areas, basically where settlements are present. This type of wind is not conducive for effective power generation; it only has a lot of worth when it accompanies moving planetary winds. In later chapters, more focus will be placed on the power of wind and effective ways to design wind turbines for optimal wind power production.

\subsection{Generator}

The basic understanding of a generator is that it converts mechanical energy to electrical energy. Generators are utilized extensively in various applications and for the most part have similarities that exist between these applications. However the few differences present is what really distinguishes a system operating on an motors. With the axial flux generator design, its operability is based on permanent magnet alternators where the concept of mag- 
nets and magnetic fields are the dominant factors in this form of generator functioning. These generators have air gap surface perpendicular to the rotating axis and the air gap generates magnetic fluxes parallel to the axis. In further chapters we will take a detailed look into their basic operation and the configuration of our design.

\subsection{Magnetic Levitation}

Also known as maglev, this phenomenon operates on the repulsion characteristics of permanent magnets. This technology has been predominantly utilized in the rail industry in the Far East to provide very fast and reliable transportation on maglev trains and with on-going research its popularity is increasingly attaining new heights. Using a pair of permanent magnets like neodymium magnets and substantial support magnetic levitation can easily be experienced. By placing these two magnets on top of each other with like polarities facing each other, the magnetic repulsion will be strong enough to keep both magnets at a distance away from each other. The force created as a result of this repulsion can be used for suspension purposes and is strong enough to balance the weight of an object depending on the threshold of the magnets. In this project, we expect to implement this technology form the purpose of achieving vertical orientation with our rotors as well as the axial flux generator.

\subsection{Power Generation}

When designing a generator it is important to have a firm grasp of the basic laws that govern its performance. In order to induce a voltage in a wire a nearby changing magnetic field must exist. The voltage induced not only depends on the magnitude of the field density but also on the coil area. The relationship between the area and field density is known as flux $(\Phi)$. The way in which this flux varies in time depends on the generator design. The axial flux generator uses the changing magnetic flux to produce a voltage. The voltage produced by each coil can be calculated using Faraday's law of induction,

$$
\mathrm{V}=-\mathrm{N}(\mathrm{dO} / \mathrm{dt})
$$

\subsubsection{Induced EMF}

In order to explain how an axial flux generator is designed the elements that produce an electromotive force or voltage must be described. An induced EMF is produced by a time varying magnetic field. Michael Faraday performed experiments with steady currents and a simple transformer in hopes of producing a voltage from a magnetic field. He discovered that a constant magnetic field would not induce a voltage but a time varying field could. This was an important discovery in what is known as electromagnetic induction, a discovery that is fundamental in the design of a generator. It is this relative motion of a magnetic field producing a voltage that allows us to be creative in the ways we produce electricity.

\subsubsection{Magnetic Flux}

The magnitude of the magnetic flux is greatest when the coil in a magnetic field is perpendicular to the field. In the design of an axial flux generator it is best to keep the coils perpendicular to the field produced by the permanent magnets. In many conventional motors a winding rotates inside a magnetic field. The number of windings is increased so that each winding is positioned close to 90 degrees to the field. Figure 4.1 illustrates this concept. In our design the angle between the coils and the field does not change, instead the field itself varies with time.

Faraday's law of induction states that the induced electromotive force is equal to the change in magnetic flux over the change in time.

$$
\mathrm{V}=-\mathrm{d} \emptyset / \mathrm{dt}
$$

\section{MAGNETIC LEVITATION}

In selecting the vertical axis concept for the wind turbine that is implemented as the power generation portion of this project, certain uniqueness corresponded to it that did not pertain to the other wind turbine designs. The characteristic that set this wind generator apart from the others is that it is fully supported and rotates about a vertical axis. This axis is vertically oriented through the center of the wind sails, which allows for a different type of rotational support rather than the conventional ball bearing system found in horizontal wind turbines.

This support is called maglev, which is based on magnetic levitation. Maglev offers a near frictionless substitute for ball bearings with little to no maintenance. The four different classes are Alnico, Ceramic, Samarium Cobalt and Neodymium Iron Boron also known Nd- Fe-B. Nd$\mathrm{Fe}-\mathrm{B}$ is the most recent addition to this commercial list of materials and at room temperature exhibits the highest properties of all of the magnetic materials. It can be seen in the B-H graph shown in Figure 5.1 that Nd-Fe-B has a very attractive magnetic characteristic, which offers high flux density operation and the ability to resist demagnetization.

This attribute will be very important because the load that will be levitated will be heavy and rotating high speeds, which will exhibit a large downward force on the axis ${ }^{[3]}$. The next factor that needs to be considered is the shape and size of the magnet which is directly related to the placement of the magnets. It seems that levitation would be most effective directly on the central axis line where, under an evenly distributed load, the wind turbine center of mass will be found as seen in Figure 5.2. This figure shows a basic rendition of how the maglev will be integrated into the design. 
If the magnets where ring shaped then they could easily be slid tandem down the shaft with the like poles facing toward each other. This would enable the repelling force required to support the weight and force of the wind turbine and minimize the amount of magnets needed to complete the concept. The permanent magnets that were chosen for this application were the N42 magnets. These are Nd-Fe-B ring shaped permanent magnets that are nickel plated to strengthen and protect the magnet itself. The dimensions for the magnets are reasonable with an outside diameter of $40 \mathrm{~mm}$, inside diameter of $20 \mathrm{~mm}$ and height of $10 \mathrm{~mm}$.

\subsection{Magnetic Selection}

Some factors need to be assessed in choosing the permanent magnet selection that would be best to implement the maglev portion of the design. Understanding the characteristics of magnet materials and the different assortment of sizes, shapes and materials is critical. There are four classes of commercialized magnets used today which are based on their material composition each having their own magnetic properties.

The four different classes are Alnico, Ceramic, Samarium Cobalt and Neodymium Iron Boron also known Nd- Fe-B. Nd-Fe-B is the most recent addition to this commercial list of materials and at room temperature exhibits the highest properties of all of the magnetic materials. It can be seen in the B-H graph shown in Figure 5.1 that Nd-Fe-B has a very attractive magnetic characteristic which offers high flux density operation and the ability to resist demagnetization. This attribute will be very important because the load that will be levitated will be heavy and rotating a high speeds which will exhibit a large downward force on the axis.

The next factor that needs to be considered is the shape and size of the magnet which is directly related to the placement of the magnets. It seems that levitation would be most effective directly on the central axis line where, under an evenly distributed load, the wind turbine center of mass will be found as seen in Figure 5.2. This figure shows a basic rendition of how the maglev will be integrated into the design. If the magnets where ring shaped then they could easily be slid tandem down the shaft with the like poles facing toward each other. This would enable the repelling force required to support the weight and force of the wind turbine and minimize the amount of magnets needed to complete the concept.

The permanent magnets that were chosen for this application were the N42 magnets. These are Nd-Fe-B ring shaped permanent magnets that are nickel plated to strengthen and protect the magnet itself. The dimensions for the magnets are reasonable with an outside diameter of $40 \mathrm{~mm}$, inside diameter of $20 \mathrm{~mm}$ and height of $10 \mathrm{~mm}$.

\section{PROTOTYPE}

Some modifications are made in order to overcome the limitations of theoretical design of magnetically levitated vertical axis wind turbine and a prototype is constructed. This section includes details of modifications made.

\subsection{Magnet Placement}

Two ring type neodymium (NdFeB) magnets of grade $\mathrm{N}$ 42 of outer diameter $40 \mathrm{~mm}$, inner diameter $20 \mathrm{~mm}$ and thickness $10 \mathrm{~mm}$ are placed at the center of the shaft by which the required levitation between the stator and the rotor is obtained. Similar disc type magnets of $30 \mathrm{~mm}$ diameter and $4 \mathrm{~mm}$ thickness are arranged as alternate poles one after the other, along the periphery of the rotor made of acrylic of $40 \mathrm{~mm}$ diameter as in Figure 6.1. These magnets are responsible for the useful flux that is going to be utilized by the power generation system.

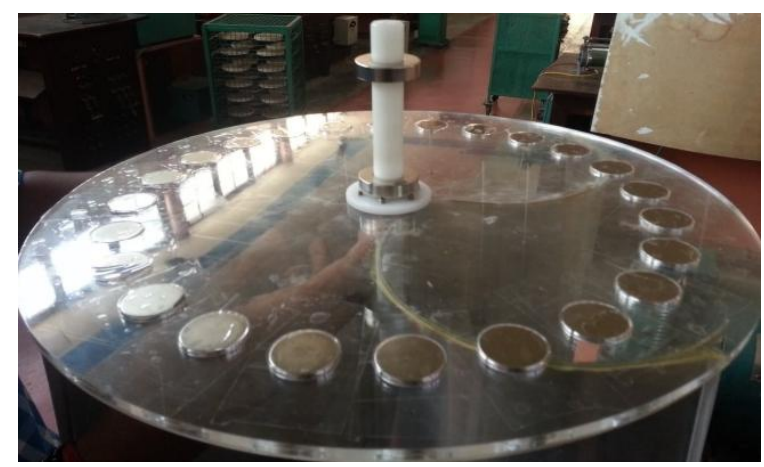

Fig 6.1 - Magnet Arrangement

\subsection{Coil Arrangement}

26 gauge wires of 1000 turns each are used as coils for power generation. 12 sets of such coils are used in the prototype. These coils are arranged in the periphery of the stator exactly in a line to the arranged disc magnets.

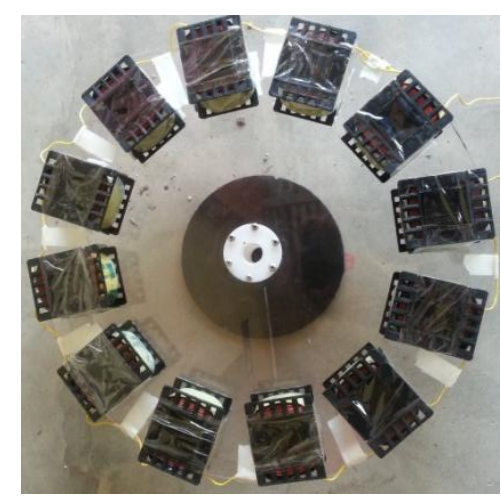

Fig 6.2 - Coil Arrangement 
The coils are raised to a certain height for maximum utilization of the magnetic flux. Each set of such coils are connected in series aiding to obtain maximum output voltage ${ }^{[4]}$. The series connections of the coils are preferred over the parallel connection for optimizing a level between the output current and voltage. The coil arrangement is shown in figure 6.2.

\subsection{Levitation between Stator \& Rotor}

In the designed prototype, the stator and rotor are separated in the air using the principle of magnetic levitation. The rotor is lifted by a certain centimeters in the air by the magnetic pull forces created by the ring type Neodymium magnets. This is the principal advantage of a maglev windmill from a conventional one. That is, as the rotor is floating in the air due to levitation, mechanical friction is totally eliminated. That makes the rotation possible in very low wind speeds. Figure 6.3 illustrates the magnetic levitation in our prototype.

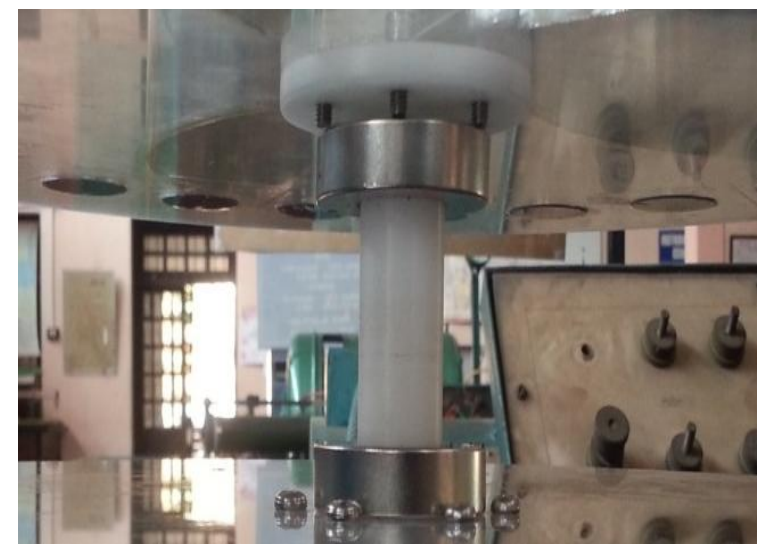

Fig 6.3 - Magnetic Levitation

\subsection{Blade Design}

The blades used in this prototype are not of the conventional type. In this prototype, As compared to the standard design model of the Savonius, we took a bit of a different approach in our design by modifying it with a curvature design from the top of the sails to the bottom ${ }^{[2]}$. This design was attained with four triangular shapes cut out from aluminum sheet metal and due to the flexibility of the sheet metal, we were able to spiral the sail from the top of the shaft to the base ${ }^{[6]}$. The height of the blades were $400 \mathrm{~mm}$.

\subsection{Final Model}

The overall structure of the prototype designed is shown in the figure 6.6. The output voltage obtained from this prototype is measured using a multimeter and a maximum of 45 volts DC were obtained.

A Led load of $3 \mathrm{~W}$ is connected to the turbine and current is measured to be 0.4 Amps.

\section{CONCLUSIONS}

At the end of the project, the magnetically levitated vertical axis wind turbine was a success. The rotors that were designed harnessed enough air to rotate at low and high wind speeds while keeping the centre of mass closer to the base yielding stability. The wind turbine rotor levitated properly using permanent magnets, which allowed for a smooth rotation with negligible friction.

Generator satisfied the specifications needed to supply the LED load. An output ranging from $40 \mathrm{~V}$ to $45 \mathrm{~V}$ was obtained from the magnetic levitated vertical axis wind turbine prototype. A modified design of savonius model wind turbine blade was used in the construction of the model. An aluminium shaft was used to avoid the wobbling movement of the rotor. Overall, the magnetic levitation wind turbine was a successful model.
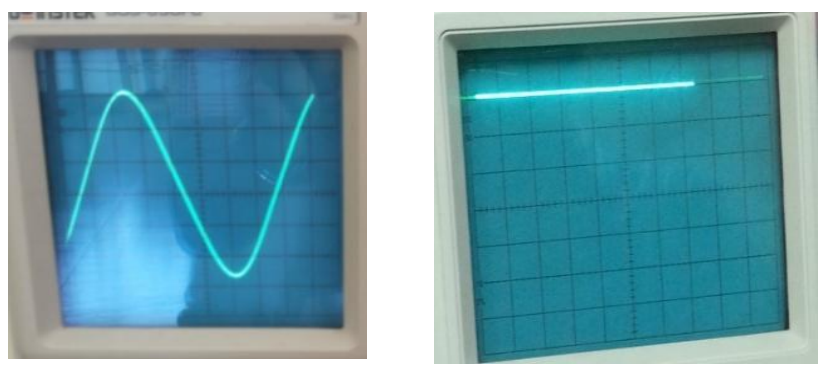

Fig. 6.6 - Output waveform before \& after rectification.

\subsection{Limitations}

In terms of large scale power production, vertical axis wind turbines have not been known to be suitable for these applications. Due to the overall structure and complexity of the of the vertical axis wind turbine, to scale it up to a size where it could provide the amount of power to satisfy a commercial park or feed into the grid would not be practical. The size of the rotors would have to be immense and would cost too much to make. Aside from the cost, this type of consumer would not desire the area that it would consume and the aesthetics of the product. Horizontal axis wind turbines are good for these applications because they do not take up as much space and are positioned high up where they can obtain higher wind speeds to provide an optimum power output.

\subsection{Future Scope}

The home for the magnetically levitated vertical axis wind turbine would be in residential areas. Here it can be mounted to a roof and be very efficient and practical. A home owner would be able to extract free clean energy thus experiencing a reduction in their utility cost and also contribute to the "Green Energy" awareness that is increasingly gaining popularity. The maglev windmill can 
be designed for using in a moderate scale power generation ranging from 400 Watts to $1 \mathrm{KW}$. Also it is suitable for integrating with the hybrid power generation units consisting of solar and other natural resources.

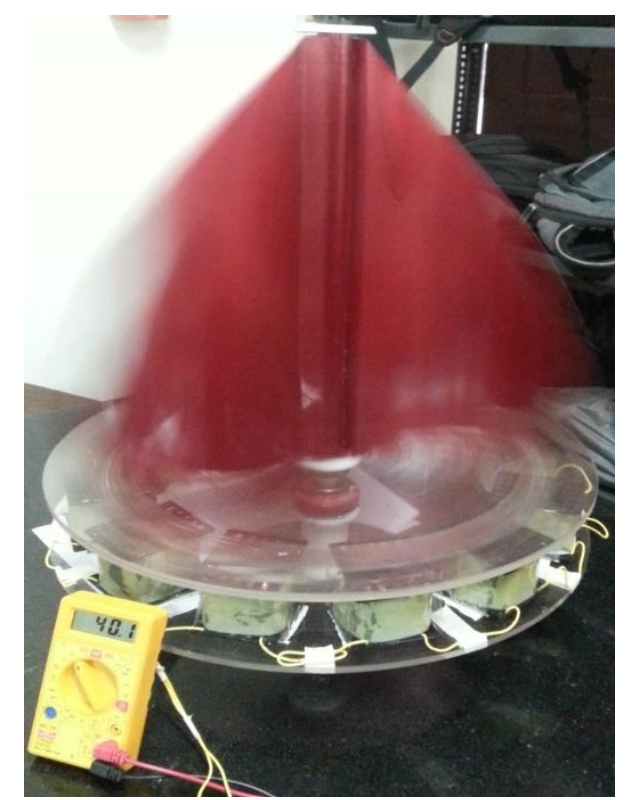

Fig 7.1 - The finished model generating a voltage of $40 \mathrm{~V}$.

\section{ACKNOWLEDGEMENTS}

I thank almighty for bestowing upon us all his blessings for the compilation of this project. I would like to express our gratitude to Dr. George Isaac, Principal, Mar Athanasius College of Engineering, Kothamangalam and Prof. K. Radhakrishnan, Head of the Department, Electrical and Electronics, for providing us with the guidance and facilities for the project. A deep sense of gratitude to project guide Prof. Rajan P Thomas, whose overall direction and guidance has been responsible for the successful completion of this project. I express our sincere gratitude to Project coordinator Prof. Elizabeth Sebastian, for her cooperation and guidance for preparing Project. I also extend our sincere thanks to all other faculty members of Electrical and Electronics Department and our friends for their support and encouragement.

\section{REFERENCES}

[1] Dinesh N Nagarkarand Dr. Z. J. Khan,"Wind Power Plant Using Magnetic Levitation Wind Turbine", InternationalJournal of Engineering and Innovative Technology (IJEIT) Volume 3, Issue 1, July 2013.

[2] Liu Shuqin,"Magnetic Suspension and Self-pitch for Vertical-axis Wind Turbines", ISBN:http://www.intechopen.com/books/fundamentaland-advanced-topics-in-wind-power/magneticsuspensionand-self-pitch-for-vertical-axis-windturbines.2011.
[3] MagLev Wind Turbine Technologies, Inc. (MWTT) \& Off Grid Technologies, Inc. (OGT)," Vertical Axis Wind Turbine 200 Mega Watt off Shore Wind Farm (VAWT Off Shore JV)-City of Evanston, Illinois Lake Michigan Project".

[4] M. A. Mueller and A. S. McDonald,"A lightweight low speed permanent magnet electrical generator for direct-drive wind turbines", Institute for Energy Systems, Institute for Energy Systems, Edinburgh University, Edinburgh University, Edinburgh, UK.

[5] Vishal D Dhareppgoaland MaheshwariM Konagutti," REGENEDYNE Maglev Wind Power Generation", SARC-IRAJ International Conference, 16th June 2013, Pune, India, ISBN: 978-8192747-8-3. 